\title{
Exfoliative Cytology of Oral Mucosa Epithelium: Cytochemical Study and Morphologic Analysis of Patients with Type 2 Diabetes
}

\author{
Yuki Ogura1, Akira Fukatsu ${ }^{2}$ \\ ${ }^{1}$ Laboratory Medicine for Dentistry, Graduate School of Dentistry at Matsudo, Nihon University, Matsudo, Japan \\ ${ }^{2}$ Department of Oral Health Science Division of Laboratory Medicine for Dentistry, Nihon University, Matsudo, Japan \\ Email: mayu16009@g.nihon-u.ac.jp
}

How to cite this paper: Ogura, Y. and Fukatsu, A. (2019) Exfoliative Cytology of Oral Mucosa Epithelium: Cytochemical Study and Morphologic Analysis of Patients with Type 2 Diabetes. Open Journal of Stomatology, 9 , 281-294.

https://doi.org/10.4236/ojst.2019.912029

Received: November 1, 2019

Accepted: December 6, 2019

Published: December 9, 2019

Copyright $\odot 2019$ by author(s) and Scientific Research Publishing Inc. This work is licensed under the Creative Commons Attribution International License (CC BY 4.0).

http://creativecommons.org/licenses/by/4.0/

(c) (i) Open Access

\begin{abstract}
Background: Diabetes mellitus is a metabolic disease characterized by chronic hyperglycemia. An association between diabetes mellitus and periodontal disease has been reported. However, few comprehensive reports have examined the impact of chronic hyperglycemia on oral mucosal epithelial cells. This study clarified the effect of diabetes on oral mucosal epithelium cells. Methods: Cells from 44 patients with type 2 diabetes and 10 healthy controls were collected from the buccal mucosa, gingiva, and lateral margin of the tongue by exfoliation. Samples were subjected to Papanicolaou staining; immunochemical staining using anti-AGE antibody, anti-Ki-67 antibody, and anti-p53 antibody; and lectin chemical staining. Furthermore, the nuclear area and cytoplasmic area were measured, and the nuclear/cytoplasm ratio was calculated. Results: A chromatin condensation-like substance was found in samples from some diabetic patients. In AGE staining, positive cells were found in both groups, although the rate of positive cells tended to be higher in diabetic patients. No positive findings were found for Ki-67 and p53 antibodies. PNA and RCA120 showed differences in staining between diabetic patients and controls. The nuclear area was significantly expanded in diabetic patients $(\mathrm{P}<0.05)$. There was no significant difference between groups in the cytoplasmic area. The nuclear/cytoplasm ratio was significantly increased in diabetic patients $(\mathrm{P}<0.05)$. Conclusion: These findings suggest that in patients with persistent hyperglycemia, changes in the sugar chain terminal of the cell surface may influence mucosal epithelial cell differentiation. The appearance of chromatin-like substances may be an indicator of the need for better management of type 2 diabetes.
\end{abstract}

\section{Keywords}

Diabetes Mellitus, Oral Exfoliative Cytology, Cytochemistry, Lectins 


\section{Introduction}

It is estimated that the total number of people with diabetes mellitus (DM) and incipient diabetes surpasses 20 million in Japan [1]. In addition, there are 420 million people with DM worldwide. It has been calculated that the worldwide population with DM will reach 700 million by 2025 if effective preventive measures are not taken [2]. DM is a metabolic disease characterized by chronic hyperglycemia in many patients; this hyperglycemia is associated with multiple complications [3]. Recently, several reports indicated that DM was associated with an increased risk for cancer [4] [5] [6] [7], including a 1.2 times increased risk for all cancers [8] [9], 1.85 times increased risk for pancreatic cancer [10], 2.5 times increased risk for liver cancer [11], and 1.30 times increased risk for colon cancer [12]. In terms of oral effects, it is reported that DM influences dry mouth, delay in healing, and periodontal disease [13] [14] [15] [16]. In addition, several researchers are conducting morphological searches for cells using exfoliative cytology, which is less invasive to patients, Seifi et al. [17] reported that the nuclear area and cytoplasmic area of oral mucosal epithelial cells in controls were significantly larger than in patients with DM. Jajarm et al. [18] reported that the nuclear and cytoplasmic areas, as well as the N/C ratio, were significantly larger in patients with DM than in controls. Alberti et al. [19] Shareef et al. [20], Sankhla et al. [21], Suvarna et al. [22], Sonawane et al. [23], and Sahu et al. [24] reported that the nuclear area and N/C ratio of oral mucosal epithelial cells were significantly increased compared with control in patients with DM, but no significant differences in the area of the cytoplasm was seen.

However, few comprehensive reports have examined the effects of DM on mucosal epithelial cells, including cell proliferation and malignant transformation.

We have been conducting morphological studies on oral mucosa using exfoliative cytology and histochemical study method [25] [26] [27] [28].

In this study, we aimed to clarify the influence of type $2 \mathrm{DM}$ on the oral mucosal epithelium by conducting cytochemical and morphologic research using exfoliative samples.

\section{Subjects and Method}

1) Subjects

A total of 44 patients (27 men and 17 women; mean age, $63.5 \pm 15.0$ years) with a diagnosis of type $2 \mathrm{DM}$ for $\geq 1$ year were included. They were diagnosed with internal medicine. Diagnostic criteria for type 2 diabetes were as follows: Plasma glucose levels (fasting $\geq 126 \mathrm{mg} / \mathrm{dl}$, 2-hour OGTT $\geq 200 \mathrm{mg} / \mathrm{dl}$, or casual $\geq 200 \mathrm{mg} / \mathrm{dl}$ ) and $\mathrm{HbAlc} \geq 6.5 \%$ [29].

The control group consisted of 10 healthy persons ( 5 men and 5 women; mean age $43.6 \pm 13.7$ years). All controls had fasting blood glucose and HbAlc NGSP (National Glycohemoglobin Standardization Program) values within the reference range. 
Subjects with anemia, other systemic diseases, oral mucosal diseases, and smokers were excluded from the study.

All subjects had good oral cleaning status, as confirmed visually, and no noticeable inflammatory symptoms. Both DM patients and controls explained this experiment and received a signed consent form.

2) Cell Collection Method

The mouth was washed, and the absence of food residues in the oral cavity was confirmed. Cells were collected from the gingival, buccal mucosa, and lateral margin of the tongue using a cytobrush (Orcellex Brush; Medical \& Biological Laboratories (MBL) Co., Ltd. Aichi, Japan).

3) Specimen Preparation

Samples were prepared by conventional cytology and liquid-based cytology (LBC) (TACAS ${ }^{\mathrm{TM}}$ method, MBL Co. Ltd., Aichi, Japan).

\section{TACAS Method Details}

Since formalin is not used for fixation, cell atrophy is mild, inflammatory cells, bacteria, necrotic cells, etc. are maintained moderately, and gene preservation is also excellent.

A special slide glass is coated with a coating agent, and the surface is positively charged. Since the cells are negatively charged, they are smeared onto the glass by attracting each other. Since there is no smearing on the part without positive charge, the sample has less cell accumulation. In addition, it has excellent hemolytic ability, and even if blood is mixed, there is little influence on observation.

a) Conventional Cytology

Oral mucosal epithelial cells were smeared on a glass slide and immersed in $95 \%$ alcohol for fixation.

b) LBC Method

Oral mucosal epithelial cells were fixed for more than 30 min with a fixative and then $1.5 \mathrm{~mL}$ per sample was transferred to a centrifuge tube and centrifuged at $2200 \mathrm{rpm}$ for $5 \mathrm{~min}$. The supernatant was removed and mixed with $3 \mathrm{~mL}$ distilled water (DW). Samples were centrifuged at $2200 \mathrm{rpm}$ for $5 \mathrm{~min}$, and the supernatant was removed again and mixed with $300 \mu \mathrm{L}$ DW. Samples $(300 \mu \mathrm{L})$ were placed on the slide, incubated for $10 \mathrm{~min}$, and rinsed with $95 \%$ ethanol. Then they were immersed in 95\% ethanol and fixed.

4) Staining Methods

a) Papanicolaou Stain

Samples prepared using conventional cytology and the LBC method were rinsed with water, immersed in $0.5 \%$ hydrochloric acid alcohol 10 to 15 times, rinsed with 95\% alcohol, and underwent OG-6 (Muto Pure Chemicals Co. Ltd. Tokyo, Japan) staining for $5 \mathrm{~min}$. Samples were then rinsed again with $95 \%$ alcohol and underwent EA-50 (Muto Pure Chemicals Co., Ltd. Tokyo, Japan) staining for $5 \mathrm{~min}$, were dehydrated in $95 \%$ and $100 \%$ alcohol, were cleared through xylene and then mounted on slides.

b) Immunocytochemical Staining (Advanced Glycation Endproducts [AGE], 
Ki-67, and p 53)

Slides prepared using the LBC method were rinsed with water. After blocking endogenous peroxidase activity with $3 \% \mathrm{H}_{2} \mathrm{O}_{2}$-methanol for $15 \mathrm{~min}$, each sample was rinsed with phosphate buffered saline (PBS) 3 times for 3 min. AGE staining was done using the microwave method $(0.01 \mathrm{M}$ citric acid buffer solution $\mathrm{pH}$ $6.0,500 \mathrm{~W}, 15 \mathrm{~min}$ ); p53 staining was done using the pressure cooker method (Tris-EDTA buffer pH 9.0, 2 min heating); and ki-67 staining was done using a boiling process (Tris-EDTA buffer $\mathrm{pH}$ 9.0, $30 \mathrm{~min}$ ), slow cooling, and rinsing each sample with PBS 3 times for 3 min.

Anti-AGE antibody (Trans Genic, Fukuoka, Japan) diluted by $2 \mu \mathrm{g} / \mathrm{ml}$, anti-p53 antibody (Dako, Glostrup, Denmark) diluted by $20 \mu \mathrm{g} / \mathrm{ml}$, and anti-Ki-67 antibody (Dako) diluted by $20 \mu \mathrm{g} / \mathrm{ml}$ were used as the primary antibodies. After 30 min incubation at room temperature, each sample was rinsed with PBS 3 times for $3 \mathrm{~min}$.

Samples were incubated at room temperature with a second antibody (DakoChemoMate Envision Kit HRPGlostrup, Denmark) for 30 min and rinsed with PBS 3 times for $3 \mathrm{~min}$.

Nuclear staining with Carrazzi's Hematoxylin was performed after color development in 3,3'-Diaminobenzidine, tetrahydrochloride (DAB; Liquid DAB + Substrate Chromogen System, Dako, Glostrup, Denmark), rinsed with water, and mounted on slides.

As a positive control, AGEs staining was used for diabetic kidney tissue, and Ki-67 and p53 staining was used for oral squamous cell carcinoma cases.

In AGEs immunocytochemical staining, 10 fields of view were observed with a $400 \times$ objective lens, and the ratio of positive cells to the observed cells of patients with DM and controls was calculated.

Judgment Criteria

AGEs were positive if the cell surface and cytoplasm were stained brown, and $\mathrm{Ki}-67$ and p53 were positive if the cell nucleus was stained brown.

c) Lectin Cytochemical Staining

Slide samples created using the LBC method were rinsed under running water. After blocking endogenous peroxidase activity with $3 \% \mathrm{H}_{2} \mathrm{O}_{2}$-methanol for 15 min, samples were rinsed with PBS 3 times for $3 \mathrm{~min}$.

Biotin lectin (ConA, SBA, WGA, DBA, UEA-I, RCA 120 , PNA (Biotinylated Lectin Kit I Vecter, Burlingame, California, USA) diluted by $20 \mu \mathrm{g} / \mathrm{ml}$ was used as the primary antibody. After $30 \mathrm{~min}$ incubation at room temperature, samples were rinsed with PBS 3 times for 3 min.

Streptavidin/HRP (Dako, Glostrup, Denmark) diluted by $2 \mu \mathrm{g} / \mathrm{ml}$ of Dako REAL $^{\mathrm{TM}}$ Antibody Diluent (Dako, Glostrup, Denmark) was used as the second antibody. After $10 \mathrm{~min}$ incubation at room temperature, samples were rinsed with PBS 3 times for 3 min. I Nuclear staining with Carrazzi's Hematoxylin after color development in DAB was done, and samples were rinsed DW and mounted on slides. 
The evaluation of staining was performed using Image $J$ (National Institutes of Health [NHI], Maryland, USA) to measure the brightness, and the following criteria were used based on 4 stages: $(-)$, negative; $( \pm)$, slightly positive; $(+)$ moderately positive; and $(++)$ strongly positive. Each luminance was defined as $(-), \geq 150 ;( \pm), 116$ to $149 ;(+), 96$ to 115 ; and $(++), \leq 95$.

5) Cytomorphologic analysis

Ten cells per field of view were extracted with a $400 \times$ magnification, and the cytoplasmic area and nuclear area were calculated with the Image J (NHI Maryland USA). The N/C ratio was calculated from these results.

For statistical analysis, the Mann-Whitney U test was used with Bonferroni's adjustment. All analyses were conducted with SPSS statistics, version 22 (IBM, Tokyo, Japan). A p value $<0.05$ was considered significant.

The Nihon University School of Dentistry at Matsudo Ethics Committee approved this research (approval number: EC17-031).

\section{Results}

1) Papanicolaou Stain

Of 44 samples from patients with DM, 23 showed strong hematoxylin staining in the nuclei. The patterns were rod-like, with incorrect margins. Substances connected to both poles in the nuclei (hereafter referred to as chromatin condensation-like substance (CCLS) are shown in (Figures 1(A)-(D)). These substances were found in both light green-positive and orange G-positive stained cells.

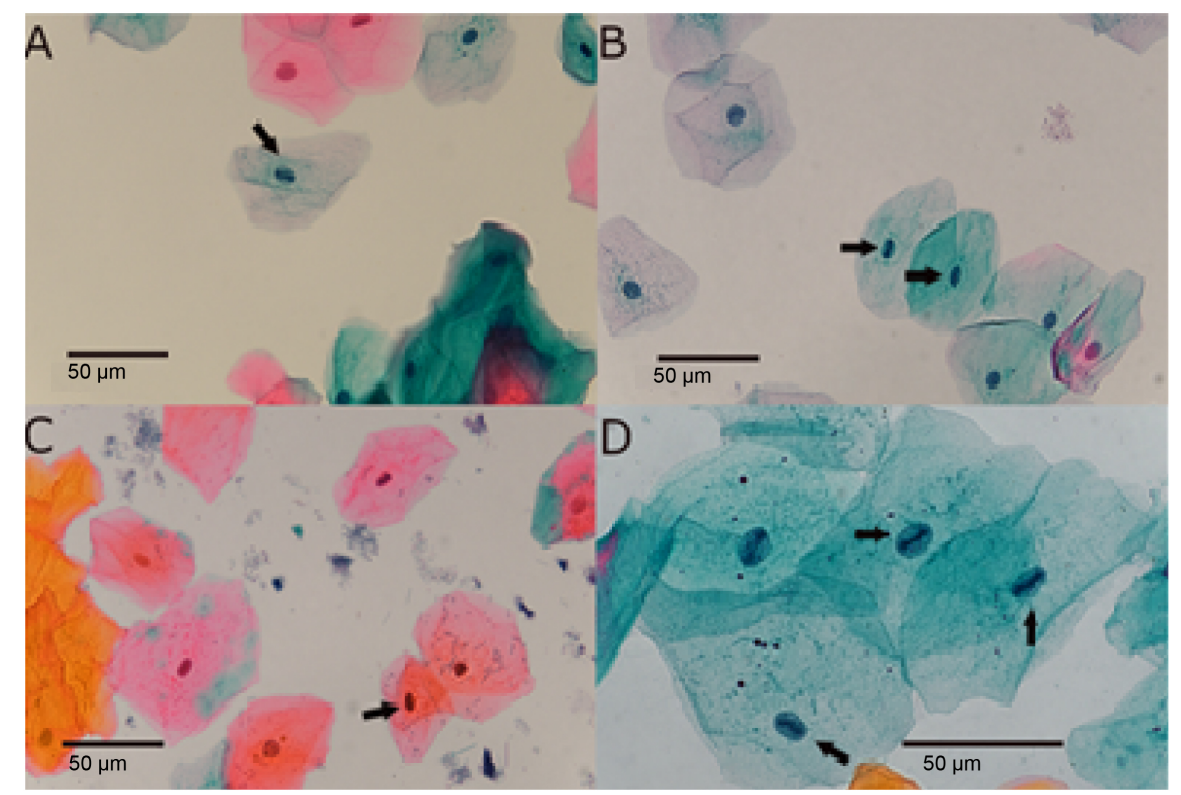

Figure 1. Papanicolaou stain. (A): buccal mucosa, (B): gingival, (C): lateral margin of the tongue, (D): gingival. A rod-like substance is finding in the cell nucleus of each sample (arrow) ((A)-(D)). A rod-like substance tends to be more in light green staining cells. A rod-like substance looks like chromatin is condensation. Magnification: $60 \times((\mathrm{A})-(\mathrm{C}))$, $100 \times(D)$. Scale bars represent $50 \mu \mathrm{m}$. 
Cells with rod-like substances in the nucleus were found in about $0.8 \%$ of gingival specimens, with a breakdown of $0.6 \%$ for light green-positive cells and $0.2 \%$ for orange G-positive cells. Cells with rod-like substances in the nucleus were found in about $0.5 \%$ of buccal specimens, with a breakdown of $0.4 \%$ for light green-positive cells and $0.1 \%$ for orange G-positive cells. Cells with rod-like substances in the nucleus were found in about $0.3 \%$ of specimens from the lateral margin of tongue with a breakdown of $0.2 \%$ for light green-positive cells and $0.1 \%$ for orange G-positive cells. Figure 1 shows the high frequency of lightgreen stained cells in all areas. The frequency of appearance by area was gingival > buccal > lateral margin of the tongue. Almost no CCCLs were found in any samples from controls (Table 1).

2) Immunocytochemical Staining

AGEs

Cells in the cytoplasm and was positive in the samples from all 3 sites in patients with DM and controls. The kidney tissue of patients with DM was used as a positive control (Figures $2(\mathrm{~A})-(\mathrm{F})$ ).

Table 1. Data from Papanicolaou staining in patients with type 2 diabetes mellitus.

\begin{tabular}{|c|c|c|c|c|c|c|c|c|c|}
\hline No. & $\begin{array}{c}\text { Buccal } \\
\text { Mucosa }\end{array}$ & Gingival & Tongue & $\begin{array}{c}\text { HbAlc \% } \\
\text { (NGSP) }\end{array}$ & No. & $\begin{array}{c}\text { Buccal } \\
\text { Mucosa }\end{array}$ & Gingival & Tongue & $\begin{array}{c}\text { HbAlc \% } \\
\text { (NGSP) }\end{array}$ \\
\hline 1 & + & + & + & 11.8 & 23 & + & - & + & 7.2 \\
\hline 2 & + & + & + & 11.3 & 24 & - & - & - & 7.1 \\
\hline 3 & + & + & + & 10.4 & 25 & - & - & - & 7.1 \\
\hline 4 & + & + & + & 10.2 & 26 & + & + & + & 7.1 \\
\hline 5 & + & + & + & 9.1 & 27 & - & - & - & 6.9 \\
\hline 6 & + & - & + & 9.1 & 28 & - & - & - & 6.9 \\
\hline 7 & + & + & - & 9.0 & 29 & - & - & - & 6.9 \\
\hline 8 & + & + & + & 8.7 & 30 & - & + & - & 6.9 \\
\hline 9 & - & + & + & 8.7 & 31 & - & - & - & 6.7 \\
\hline 10 & + & + & + & 8.3 & 32 & - & - & - & 6.5 \\
\hline 11 & + & + & + & 8.0 & 33 & - & - & - & 6.4 \\
\hline 12 & + & + & + & 7.9 & 34 & - & - & - & 6.4 \\
\hline 13 & + & - & - & 7.8 & 35 & - & - & - & 6.3 \\
\hline 14 & - & - & - & 7.7 & 36 & - & - & - & 6.3 \\
\hline 15 & + & + & + & 7.7 & 37 & - & - & - & 6.1 \\
\hline 16 & + & + & + & 7.6 & 38 & + & + & + & 6.1 \\
\hline 17 & + & + & + & 7.6 & 39 & - & - & - & 5.9 \\
\hline 18 & + & + & + & 7.5 & 40 & - & - & - & 5.9 \\
\hline 19 & + & + & + & 7.4 & 41 & - & - & - & 5.8 \\
\hline 20 & + & + & + & 7.3 & 42 & - & - & - & 5.8 \\
\hline 21 & - & - & - & 7.3 & 43 & - & - & - & 5.6 \\
\hline 22 & - & - & - & 7.3 & 44 & - & - & - & 5.2 \\
\hline
\end{tabular}

+: Chromatin with condensation-like substance; -: chromatin without condensation-like substance. 


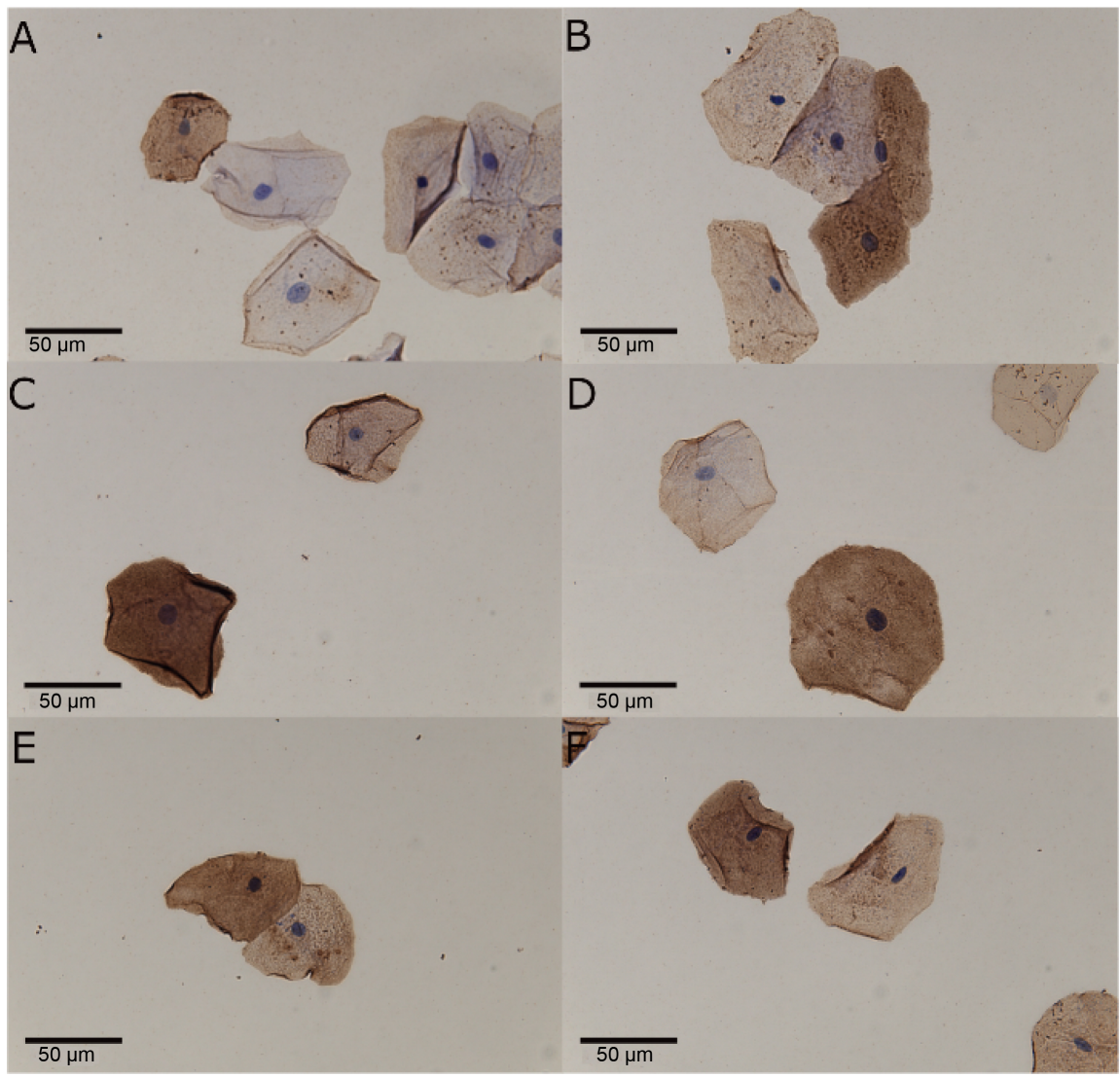

Figure 2. AGE stain. (A): DM gingival, (B): control gingival, (C): DM buccal mucosa, (D): control buccal mucosa, (E): DM lateral margin of the tongue, (F): control lateral margin of the tongue. Positive reactions were observed in the cytoplasm and cytoplasm margin of the specimens of DM patients and control (A)-(F). There are more positive cells in DM compared to control. Magnification: 60× (A)-(F). Scale bars represent $50 \mu \mathrm{m}$.

The percentage of positive cells at each site was as follows: gingival area, $30 \%$ for patients with DM and 17\% for controls; buccal area, $32 \%$ for patients with $\mathrm{DM}$ and $21 \%$ for controls; lateral margin of tongue, $26 \%$ for patients with DM and $14 \%$ for controls. The proportion of positive cells tended to be higher in patients with DM than in controls.

$\underline{\mathrm{Ki}-67}$

No positive findings were found in the cell nuclei of patients with DM or controls.

The positive control was positive in the nuclear in cases of OSCC (oral squamous cell carcinoma).

p53

No positive findings were found in the cell nuclei of patients with DM or controls.

The positive control was positive in the nuclear in cases of OSCC.

3) Lectin cytochemical staining

ConA

Negative or slightly positive findings were seen in the cytoplasm of patients 
with DM and controls.

$\underline{\text { SBA }}$

Moderate to strongly positive findings were seen in the cytoplasm of patients with DM. Slight to moderately positive findings were seen in the cytoplasm of controls.

WGA

Moderate to strongly positive findings were seen in the cytoplasm of patients with DM and controls.

\section{$\underline{\mathrm{DBA}}$}

Negative findings were seen in the cytoplasm of patients with DM. Negative to moderately positive findings were seen in the cytoplasm of controls.

\section{UEA-I}

Negative to slightly positive findings were seen in the cytoplasm of patients with DM and controls.

$\underline{\mathrm{RCA}_{120}}$

Moderate to strongly positive findings were seen in the cytoplasm of patients with DM. Negative to moderately positive findings were seen in the cytoplasm of controls.

PNA

Moderate to strongly positive findings were seen in the cytoplasm of patients with DM. Slight to moderately positive findings were seen in the cytoplasm of controls.

PNA and RCA120 showed differences in lectin between patients with DM and controls (Figures 3(A)-(D) and Table 2).

4) Cell Morphologic Analysis

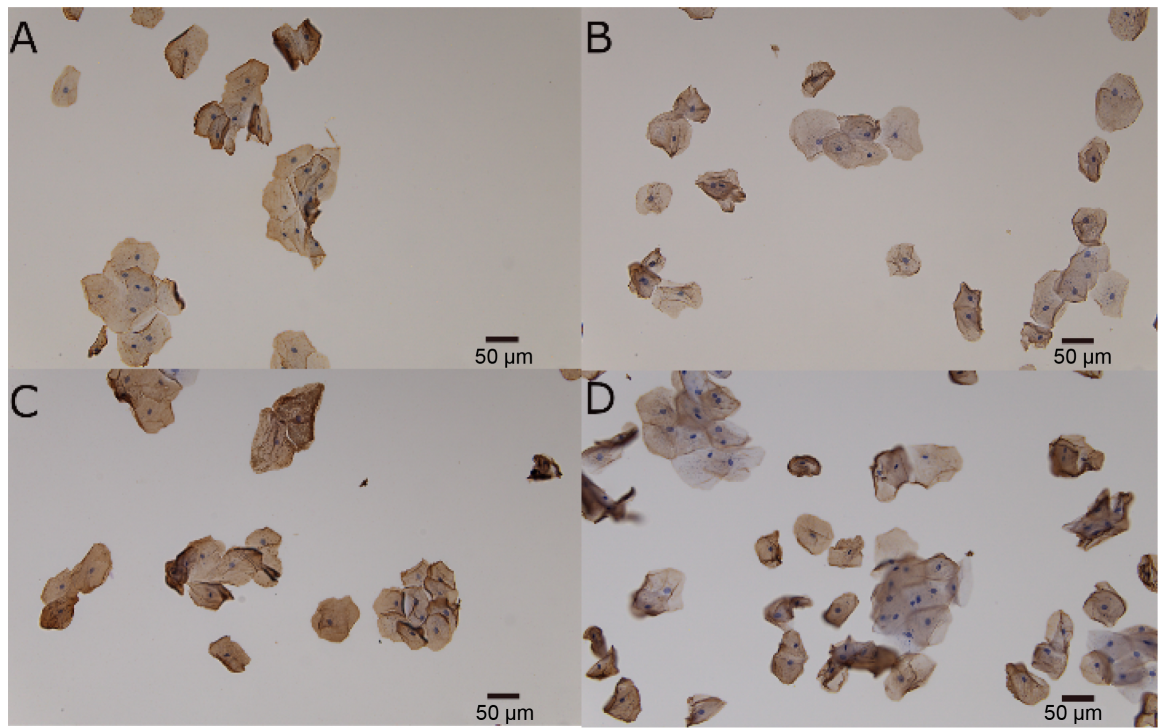

Figure 3. Lectin stain. (A): $\mathrm{DM} \mathrm{RCA}_{120}$, (B): control $\mathrm{RCA}_{120},(\mathrm{C})$ : DM PNA, (D): control PNA. RCA R $_{120}$ show staining to cytoplasm ((A), (B)). PNA show staining to cytoplasm $((C),(D))$. There was a difference in staining intensity in both stains, DM showed more staining. Magnification: $20 \times((\mathrm{A})-(\mathrm{D}))$. Scale bars represent $50 \mu \mathrm{m}$. 
In terms of the nuclear area and the cytoplasmic areas, the nuclear area increased significantly in patients with DM. There were no significant differences between groups in the cytoplasmic area. The N/C ratio was significantly increased in patients with DM. There were no significant differences between groups in terms of CCLS (+) and CCLS (-) (Figures 4-6 and Table 3).

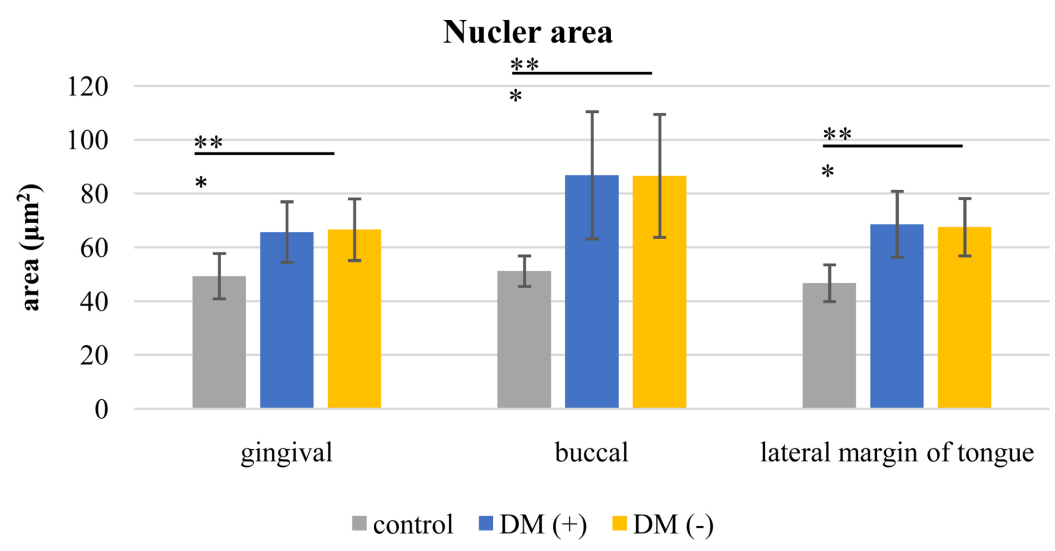

Figure 4. Comparison of nuclear area between $\mathrm{DM}(+), \mathrm{DM}(-)$ and control. ${ }^{*} \mathrm{p}<$ 0.05 (vs $\mathrm{DM}(+))$ ), ${ }^{* *} \mathrm{p}<0.05$ (vs $\left.\mathrm{DM}(-)\right)$. DM(+): chromatin with condensation-like substance, DM(-): Chromatin without condensation-like substance.

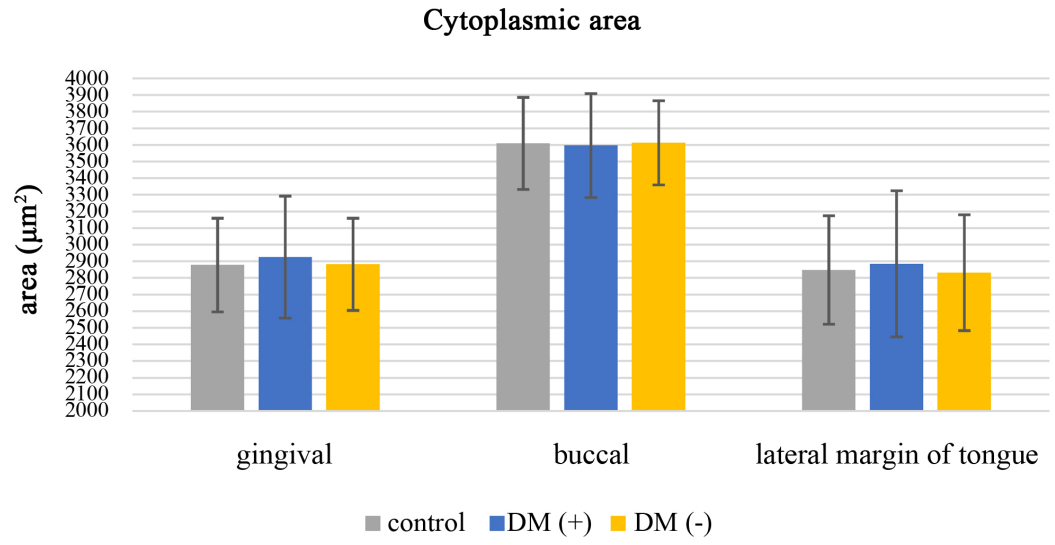

Figure 5. Comparison of cytoplasmic area between $\mathrm{DM}(+), \mathrm{DM}(-)$ and control. NS $\mathrm{p}<0.05$.

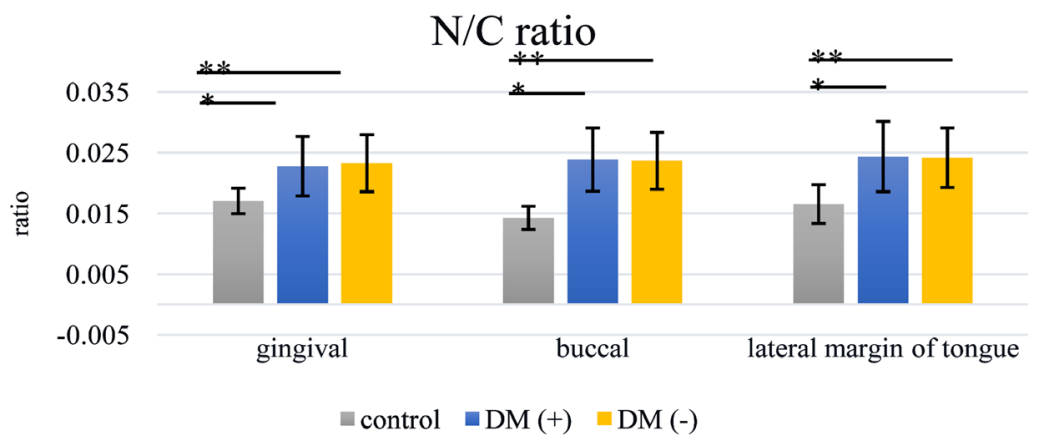

Figure 6. Comparison of N/C ratio between $\mathrm{DM}(+), \mathrm{DM}(-)$ and control. ${ }^{*} \mathrm{p}<0.05$ (vs $\mathrm{DM}(+)),{ }^{* *} \mathrm{p}<0.05$ (vs $\left.\mathrm{DM}(-)\right)$. 
Table 2. Lectin staining results.

\begin{tabular}{ccc}
\hline Lectin & Patients with Type 2 Diabetes & Controls \\
\hline ConA & - to \pm & - to \pm \\
SBA & + to ++ & \pm to + \\
WGA & + to ++ & + to ++ \\
DBA & - & - to + \\
UEA-1 & - to \pm & - to \pm \\
RCA 120 & + to ++ & - to + \\
PNA & + to ++ & \pm to + \\
\hline
\end{tabular}

$(-)$, negative; ( \pm ), slightly positive; (+), moderately positive; $(++)$, strongly positive.

Table 3. Imag J result of nucler area, cytoplasmic area and nucler/cytoplasmic ratio.

\begin{tabular}{ccccc}
\hline \multirow{2}{*}{ Detemination } & & $\mathrm{DM}(+)$ & $\mathrm{DM}(-)$ & Control Group \\
\cline { 3 - 5 } & & Mean $\pm \mathrm{SD}$ & Mean $\pm \mathrm{SD}$ & Mean $\pm \mathrm{SD}$ \\
\hline \multirow{3}{*}{ Nucler Area $\left(\mu \mathrm{m}^{2}\right)$} & gingival & $65.68 \pm 11.28$ & $66.58 \pm 11.44$ & $49.34 \pm 8.42$ \\
& buccal & $86.84 \pm 23.65$ & $86.59 \pm 22.83$ & $51.18 \pm 5.70$ \\
& tongue & $68.60 \pm 12.27$ & $67.51 \pm 10.59$ & $46.70 \pm 6.80$ \\
& gingival & $2926.49 \pm 366.33$ & $2882.70 \pm 277.53$ & $2878.35 \pm 281.72$ \\
Cytoplasmic Area $\left(\mu \mathrm{m}^{2}\right)$ & buccal & $3596.45 \pm 312.48$ & $3612.72 \pm 252.61$ & $3609.48 \pm 277.05$ \\
& tongue & $2885.58 \pm 439.82$ & $2832.53 \pm 348.29$ & $2848.38 \pm 325.69$ \\
& gingival & $0.0228 \pm 0.0049$ & $0.0233 \pm 0.0047$ & $0.0171 \pm 0.0021$ \\
Nucler/Cytoplasmic Ratio & buccal & $0.0239 \pm 0.0052$ & $0.0237 \pm 0.0047$ & $0.0143 \pm 0.0019$ \\
& tongue & $0.0244 \pm 0.0058$ & $0.0242 \pm 0.0049$ & $0.0166 \pm 0.0032$ \\
\hline
\end{tabular}

$\mathrm{DM}(+)$ : Chromatin condensation-like substance having, $\mathrm{DM}(-)$ : chromatin condensation-like substance not having.

\section{Discussion}

Papanicolaou staining showed that when cells of patients with DM were compared with cells of control subjects, CCLS was observed in the cell nuclei of patients with DM. It is well known that chromatin condensation is associated with cell proliferation [30]. Cell division can be observed only in the basal cell layer in the oral mucosal epithelium. Cells in the vicinity of basal cells cannot be collected by exfoliative cytology. In this study, CCLS were found in epithelial cells in the surface layer system. Based on this finding, it was suspected that these cells might be in the active proliferating stage.

Recently, the association of cancer risk has been reported by diabetes [8] [9] [10] [11] [12]. Therefore, it was necessary to examine the cell proliferating ability and malignant transformation ability of CCLS. Immunochemical studies were conducted using Ki-67, which is an indicator of cell proliferation ability, and p53, a tumor suppressor gene whose expression is observed during malignant transformation. Both Ki-67 and p53 studies showed negative findings. Based on these findings, the observed cells did not show an increase in cell proliferation ability or any malignant transformation. In addition, when cell division occurs, 
not only chromatin condensation, but also nuclear membrane loss occurs. The correlation with the cell cycle is considered to be low, considering the clearness of the nuclear membrane of cells with CCLS found in this study.

When the oral mucous membrane epithelial cells of patients with DM and controls were compared morphologically, the nuclear area and the N/C ratio was significantly larger in patients with DM compared to controls. The results of this study were similar to those of Alberti et al. [19], Shareef et al. [20], Sankhla et al. [21], Suvarna et al. [22], Sonawane et al. [23], and Sa-hu et al. [24]. Only nucleus increases, referred to nuclear swelling, is found in cells affected by the proliferation phase, cells affected by inflammation, and tumor cells. As a result of immunocytochemical staining of cells in this study, there was no possibility of enhancement of cell proliferation ability or malignant transformation. Therefore, it is considered that the nuclear swelling observed in the epithelial cells of the oral mucosa in patients with DM was not related to cell proliferation or malignant transformation.

In addition, because there were no clinical signs of inflammation in the oral cavity of the subjects, and the appearance of inflammatory cells was rarely observed in Papanicolaou-stained specimens of exfoliative cytology samples, it is assumed that this change was not attributable inflammation.

The appearance of CCLS in the nucleus was mainly observed in the oral mucosal epithelial cells of patients with DM with a high HbA1c (NGSP) level $(\geq 7.0 \%)$. It was assumed that the appearance of CCLS may be affected by sustained hyperglycemia. Therefore, we performed anti-AGE antibody in for samples of DM patients.

AGEs are produced when sugars in the blood, such as glucose, and proteins undergo a glycation reaction (Maillard reaction). In DM, AGEs accumulate in the Renal tube, retinas capillary, blood vessels wall, and the like. These AGEs cause an inflammatory response by inducing oxidative stress and increasing the expression of inflammatory cytokines.

It was reported that patients with DM had higher rates of AGEs-positive cells in the gingival epithelium compared to controls [31]. In this study, the incidence of AGEs-positive cells tended to be higher, although differences did not reach statistical significance in patients with DM in all parts of the gums, the buccal mucosa, and the tongue. These results were the same as the report by Zizzi et al. [32]. However, it cannot be denied that CCLS seen in this study may be due to increased oxidative stress caused by the accumulation of AGEs.

To investigate the influence of hyperglycemia, we further examined the binding of the sugar chain end using lectin cell chemistry. Lectins are proteins that bind to specific carbohydrate structures. Many kinds of lectins are isolated from plants, animals, and microorganisms. It is well known that changes in lectin binding patterns are related to cell differentiation and malignant transformation [33] [34]. In this study, 7 types of lectins, ConA, SBA, WGA, DBA, UEA-I, RCA120, and PNA, were used. A clear difference in staining was observed be- 
tween patients with DM and controls in 2 types of lectins, PNA and RCA120. This finding suggests that there was some change in Gal- $\beta(1 \rightarrow 3)-3$ GalNAc and $\mathrm{D}-\mathrm{Gal}$ on the oral mucosa epithelial cells of patients with DM. As discussed above, sustained hyperglycemia is the most probable cause of this finding.

In Papanicolaou staining, CCLS appeared frequently in light-green adherent cells in all sites. From this finding, it is considered that cells with weak keratinization tend to be susceptible to hyperglycemic effects. In addition, it is assumed that the cause of the abnormality in the nucleus was also continuous exposure to the hyperglycemic state in many patients, which promotes glycation and causes changes in the sugar chain end of the oral mucosal epithelial cell surface. These results have some influence on cell differentiation.

The results observed in this study were suggested to be useful as a supplementary tool for determining the pathology of DM.

\section{Acknowledgements}

The authors would like to thank Professor M. Fukumoto from Nihon University School of Dentistry at Matsudo, helpful discussions and comments on the manuscript.

We wish to thank M. Suemitsu technical assistance.

\section{Conflicts of Interest}

The authors declare that there is no conflict of interest.

\section{References}

[1] Morimoto, A., Nishimura, R. and Tajima, N. (2010) Trends in the Epidemiology of Patients with Diabetes in Japan. JMA Journal, 53, 36-40.

[2] World Health Organization (2016) World Health Day 2016: Beat Diabetes. https://www.who.int/campaigns/world-health-day/2016/en

[3] Tripathi, B.K. and Srivastava, A.K. (2006) Diabetes Mellitus: Complications and Therapeutic. Medical Science Monitor, 12, RA130-47.

[4] Shikata, K., Ninomiy, T. and Kiyohara, Y. (2012) Diabetes Mellitus and Cancer Risk: Review of the Epidemiological Evidence. Cancer Science, 104, 9-14. https://doi.org/10.1111/cas.12043

[5] Shlomai, G., Neel, B., Le Roith, D. and Gallagher, E.J. (2016) Type 2 Diabetes Mellitus and Cancer: The Role of Pharmacotherapy. Journal of Clinical Oncology, 34, 4261-4269. https://doi.org/10.1200/JCO.2016.67.4044

[6] Sasazuki, S., Charvat, H., Hara, A., Wakai, K. and Nagata, C. (2013) Diabetes Mellitus and Cancer Risk: Pooled Analysis of Eight Cohort Studies in Japan. Cancer Science, 104, 1499-1507. https://doi.org/10.1111/cas.12241

[7] Kasuga, M., Ueki, K., Tajima, N., Noda, M. and Ohashi, K. (2013) Report of the JDS/JCA Joint Committee on Diabetes and Cancer. Diabetology International, 4, 8196. https://doi.org/10.1007/s13340-013-0121-3

[8] Harding, J.L., Shaw, J.E., Peeters, A., Cartensen, B. and Magliano, D.J. (2015) Cancer Risk among People with Type 1 and Type 2 Diabetes: Disentangling True Associations, Detection Bias, and Reverse Causation. Diabetes Care, 38, 264-270. 
https://doi.org/10.2337/dc14-1996

[9] Inoue, M., Iwasaki, M., Otani, T., Sasazuki, S. and Noda, M. (2006) Diabetes Mellitus and the Risk of Cancer: Results from a Large-Scale Population-Based Cohort Study in Japan. Archives of Internal Medicine, 166, 1871-1877. https://doi.org/10.1001/archinte.166.17.1871

[10] Huxley, R., Ansary-Moghaddam, A., Berrington de Gonzalez, A., Barzi, F. and Woodward, M. (2005) Type-II Diabetes and Pancreatic Cancer: A Meta-Analysis of 36 Studies. British Journal of Cancer, 92, 2076-2083.

https://doi.org/10.1038/sj.bjc.6602619

[11] El-Serag, H.B., Hampel, H. and Javadi, F. (2006) The Association between Diabetes and Hepatocellular Carcinoma: A Systematic Review of Epidemiologic Evidence. Clinical Gastroenterology and Hepatology, 4, 369-380.

https://doi.org/10.1016/j.cgh.2005.12.007

[12] Larsson, S.C., Orsini, N. and Wolk, A. (2005) Diabetes Mellitus and Risk of Colorectal Cancer: A Meta-Analysis. Journal of the National Cancer Institute, 97, 1679-1687. https://doi.org/10.1093/jnci/dji375

[13] Stanko, P. and Holla, L.I. (2014) Bidirectional Association between Diabetes Mellitus and Inflammatory Periodontal Disease. Biomedical Papers, 158, 35-38. https://doi.org/10.5507/bp.2014.005

[14] Chávarry, N.G.M., Vettore, M.V., Sansone, C. and Sheiham, A. (2009) The Relationship between Diabetes Mellitus and Destructive Periodontal Disease: A Meta-Analysis. Oral Health and Preventive Dentistry, 7, 107-127.

[15] Preshaw, P.M., Alba, A.L., Herrera, D., Jepsen, S. and Konstantinidis, A. (2012) Periodontitis and Diabetes: A Two-Way Relationship. Diabetologia, 55, 21-31. https://doi.org/10.1007/s00125-011-2342-y

[16] Seshima, F., Nishina, M., Namba, T. and Saito, A. (2016) Periodontal Regenerative Therapy in Patient with Chronic Periodontitis and Type 2 Diabetes Mellitus: A Case Report. The Bulletin of Tokyo Dental College, 57, 97-104. https://doi.org/10.2209/tdcpublication.2015-0041

[17] Seifi, S., Feizi, F., Moazzezi, Z., Mehdizadeh, M. and Zamaniet, B. (2014) Evaluation of Oral Mucosal Epithelium in Diabetic Male Patients by Exfoliative Cytology Method. Journal of Diabetes \& Metabolic Disorders, 13, 77. https://doi.org/10.1186/2251-6581-13-77

[18] Jajarm, H.H., Mohtasham, N. and Rangiani, A. (2008) Evaluation of Oral Mucosa Epithelium in Type II Diabetic Patients by an Exfoliative Cytology Method. Journal of Oral Science, 50, 335-340. https://doi.org/10.2334/josnusd.50.335

[19] Alberti, S., Spadella, C.T., Francischone, T.R.C.G., Assis, G.F. and Cestari, T.M. (2003) Exfoliative Cytology of the Oral Mucosa in Type II Diabetic Patients: Morphology and Cytomorphometry. Journal of Oral Pathology \& Medicine, 32, 538-543. https://doi.org/10.1034/j.1600-0714.2003.00162.x

[20] Shareef, B.T., Ang, K.T. and Naik, V.R. (2008) Qualitative and Quantitative Exfoliative Cytology of Normal Oral Mucosa in Type 2 Diabetic Patients. Medicina Oral Patologia Oral y Cirugia Bucal, 13, E693-E696.

[21] Sankhla, B., Shah, S., Madhusudhan, A.S. and Bhagatet, P. (2010) Exfoliative Cytology of Oral Mucosa: Cytomorphometric Analysis of Diabetic Patients. Journal of Oral Science, 2, 4-12.

[22] Suvarna, M., Anuradha, C., Kiran Kumar, K., Sekhar, P.C., Chandra, K.L.P. and Reddyet, B.V.R. (2012) Cytomorphometric Analysis of Exfoliative Buccal Cells in 
Type II Diabetic Patients. Journal of Dr. NTR University of Health Sciences, 1, 33-37. https://doi.org/10.4103/2277-8632.94173

[23] Sonawane, K., Jain, S., Gupta, I., Karthik, B.V., Singaraju, S. and Singaraju, M. (2011) Cytomorphometric Analysis of Oral Mucosa in Diabetic Patients in Bhopal Region an In-Situ Study. International Journal of Clinical Dental Science, 2, 12-15.

[24] Sahu, M., Suryawanshi, H., Nayak, S. and Kumar, P. (2017) Cytomorphometric Analysis of Gingival Epithelium and Buccal Mucosa Cells in Type 2 Diabetes Mellitus Patients. Journal of Oral and Maxillofacial Pathology, 21, 224-228. https://doi.org/10.4103/jomfp.JOMFP_152_16

[25] Sun, Y., Kuyama, K., Fukumoto, M., Nishimura, H., Burkhardt, A. and Yamamoto, H. (2011) The Sequential Changes of Carcinoma Cuniculatum: A Case Report with 11-Year Follow-Up. Journal of Oral Pathology \& Medicine, 15, 113-116. https://doi.org/10.3353/omp.15.113

[26] Kuyama, K., Matsumoto, T., Morikawa, M., Fukatsu, A., Ichimura, M., Wakami, M., Fukumoto, M., Kato, T. and Yamamoto, H. (2013) Morphometrical Findings among Dysplasias of Oral, Cervical and Bronchial Regions. Open Journal of Stomatology, 13, 215-222. https://doi.org/10.4236/ojst.2013.32037

[27] Fukatsu, A., Kuyama, K., Fuchigami, M. and Fukumoto, M. (2015) Comparison of Liquid Based Cytology and Conventional Cytology in Squamous Cell Carcinoma. Journal of the Japan Academy of Dental Human Dock, 10, 41-48.

[28] Fuchigami, M., Suemitsu, M., Fukatsu, A., Komine, C., Konish, Y., Morikawa, M., Wakami, M., Kuyama, K. and Fukumoto, M. (2016) Neutrophil Elastate, CD68, HDL-DR and CD105 Immunohistochemical Expression of Oral Verruciform Xanthoma. Journal of Japanese Society for Evidence and the Dental Professional, 8, 16-21.

[29] Seino, Y., Nanjo, K., Tajima, N., Kadowaki, T., Kashiwagi, A. and Araki, E. (2010) Report of the Committee on the Classification and Diagnostic Criteria of Diabetes Mellitus the Committee of the Japan Diabetes Society on the Diagnostic Criteria of Diabetes Mellitus. Diabetology International, 1, 2-20. https://doi.org/10.1007/s13340-010-0006-7

[30] Antonin, W. and Neumann, H. (2016) Chromosome Condensation and Decondensation during Mitosis. Current Opinion in Cell Biology, 40, 15-22. https://doi.org/10.1016/j.ceb.2016.01.013

[31] Schmidt, A.M., Weidman, E., Lalla, E., Yan, S.D., Hori, O., Cao, R., Brett, J.G. and Lamster, I.B. (1996) Advanced Glycation Endproducts (AGEs) Induce Oxidant Stress in the Gingiva: A Potential Mechanism Underlying Accelerated Periodontal Disease Associated with Diabetes. Journal of Periodontal Research, 31, 508-515. https://doi.org/10.1111/j.1600-0765.1996.tb01417.x

[32] Zizzi, A., Tirabassi, G., Aspriello, S.D., Piemontese, M., Rubini, C. and Lucarini, G. (2013) Gingival Advanced Glycation End-Products in Diabetes Mellitus-Associated Chronic Periodontitis: An Immunohistochemical Study. Journal of Periodontal Research, 48, 293-301. https://doi.org/10.1111/jre.12007

[33] Duncan, D.A., Mazur, M.T. and Younger, J.B. (1988) Glycoconjugate Distribution in Normal Human Endometrium: A Lectin Histochemical Study. International Journal of Gynecological Pathology, 7, 236-248. https://doi.org/10.1097/00004347-198809000-00004

[34] Bopp, S., EI-Hifnawi, E.-S. and Laqua, H. (1992) Lectin Binding Pattern in Human Retinal Pigment Epithelium. Annals of Anatomy, 174, 279-285.

https://doi.org/10.1016/S0940-9602(11)80281-5 